\title{
Exploring the Feasibility of an Educational Computer Game as a Novel Means of Assessing Problem Solving Competencies
}

\section{Dr. Jacob R Grohs, Virginia Tech}

Jacob Grohs is an Assistant Professor in Engineering Education at Virginia Tech with Affiliate Faculty status in Biomedical Engineering and Mechanics and the Learning Sciences and Technologies at Virginia Tech. He holds degrees in Engineering Mechanics (BS, MS) and in Educational Psychology (MAEd, $\mathrm{PhD})$.

\section{Darren K Maczka, Virginia Tech Department of Engineering Education}

Darren Maczka is a Ph.D. student in Engineering Education at Virginia Tech. His background is in control systems engineering and information systems design and he received his B.S. in Computer Systems Engineering from The University of Massachusetts at Amherst. He has several years of experience teaching and developing curricula in the department of Electrical and Computer Engineering at Virginia Tech.

\section{Ms. Michelle Soledad, Virginia Tech}

Michelle Soledad is a doctoral student and graduate research assistant in the Department of Engineering Education at the Virginia Polytechnic Institute and State University. Her research interests include faculty motivation and development towards supporting student success. Ms. Soledad has bachelor's and master's degrees in Electrical Engineering from the Ateneo de Davao University in Davao City, Philippines, where she continues to be a faculty member of the Electrical Engineering Department. She also served as Department Chair and was a member of the University Research Council before pursuing doctoral studies at Virginia Tech. Prior to joining the University in 2008, Ms. Soledad was a Senior Team Lead for Accenture, where she worked on and managed systems maintenance and enhancement projects.

\section{Miss Kiran Kelsey Bagalkotkar}




\section{Exploring the Feasibility of an Educational Computer Game as Novel Means of Assessing Problem Solving Competencies}

\section{Introduction}

This paper is grounded in the notion that educating problem solvers is one of the most critical functions of higher education. Indeed, governmental bodies and industry regularly demand such problem-solving competencies and they are becoming an increasingly intentional aspect of engineering education curricula. ${ }^{1 / 2}$ Despite the focus on problem-solving, a disconnect exists between the types of problems solved in educational settings and those encountered in professional settings and communities. ${ }^{3}$ We believe this disconnect is in part because education systems are structured on assessment (e.g., grades, diplomas, accreditation) and thus do not prioritize teaching what cannot reliably be assessed. Operationalizing and rigorously assessing complex problem solving skills requires examining the reasoning process itself in addition to evaluating the accuracy of solutions generated. Most methods of researching problem-solving process involve self-report data which, while validated, still have limitations associated with the difference between individual perception and observable action. Building on the work of others to address such limitations, ${ }^{45}$ we prioritize research protocols that move beyond pairing of achievement (right/wrong answers) and self-reported reasoning process (think-aloud protocols) to include observable data during problem-solving process that can corroborate (or refute) the self-report data.

The central aim of this paper is to explore the feasibility of using an educational computer game as a novel means of assessing problem-solving competency. In this case, the novelty of the approach compared to traditional methods is twofold: (1) the use of a computer game enables us to directly observe problem-solving process through action in the game and (2) because of the naturally immersive game environment, we hope to see motivation and persistence in the face of complex problems that might otherwise be difficult to achieve in a laboratory setting. As a feasibility study, we will present methods and data that we suggest should guide further research but should not be used to make specific claims. Before describing this exploratory study, we briefly review relevant literature of problems and problem-solving.

\section{Background on Problem Solving Literature}

One characterizing difference between problems faced in formal education compared to the professional world is described in problem-solving literature as the degree to which a problem is 
structured. Well-structured problems have clear goals which can be obtained through repeatable algorithms to get absolute correct solutions $\frac{677}{}$ In contrast, ill-structured problems are inherently vague and can have many or no absolute solution paths where solving the problem largely requires subjective arguments that frame and guide the attempted solutions. ${ }^{6 / 8}$ Traditionally, we could hope that by teaching through classical well-defined problems we provide foundational knowledge that enables students to draw upon that knowledge when solving more realistic, complex, and vague problems. However, research suggests that successful performance on well-structured problems is not a predictor of success on ill-structured problems because fundamentally different reasoning skills are needed. ${ }^{[7}$ While well-structured problems are often solved once an appropriate algorithm has been identified and used, solving ill-structured problems involves skills such as argumentation and reflective design to robustly identify the problem itself before considering potential solutions. ${ }^{910}$ Success in the setting and solving of ill-structured problems has been linked to metacognitive strategies, ${ }^{799}$ specific cognitive skills such as analogical reasoning, $\frac{11}{11}$ and epistemological beliefs. $\stackrel{12 \mid 13}{ }$ Analogical reasoning is a higher order thinking process whereby novel problems are interpreted as an amalgam of structurally similar previously solved problems. Research on differences between experts and novices in a domain further shows that the discerning choice of which analogies to draw and use is of particular importance - surface similarities alone can be misleading and experts draw upon commonalities of deeper structural similarities to aid in solving problems.

Studying problem-solving process instead of product is challenging. Use of metacognitive strategies during problem-solving has classically been studied in-situ using think-aloud

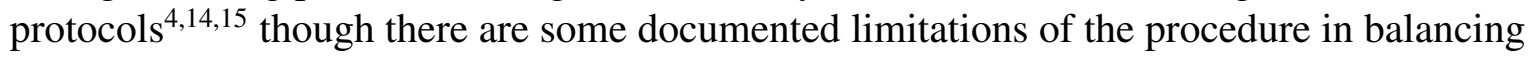
obtaining rich, meaningful data without significantly disrupting the participant's thought process itself. Psychometrically validated instruments (e.g., the Critical Thinking Dispositions Scale ${ }^{16}$ ) can also be used but are limited due to their reliance on self-report Likert-scale item response separate from a specific problem-solving context (in contrast to using an anchoring problem exercise in a think-aloud protocol). Similarly, isolating and studying analogical reasoning as a construct is challenging, in part because many problems requiring its use are either so general they seem unmoored from context or require such significant domain specific content knowledge that interpretation is difficult (e.g., Duncker's radiation problem ${ }^{17}$ ). Yet another barrier in studying problem-solving in ill-structured contexts is that it requires research participants willing to fully apply themselves to solve complex problems which may be confusing and frustrating.

This study seeks to address these various challenges by capitalizing on the appealing and immersive aspects of computer games in order to explore the feasibility of using an educational game called Contraption Maker as a problem-solving research tool. Using games for learning and novel assessment is a current area of emergent interest. ${ }^{18 / 19 \mid 20}$ Contraption Maker is a commercial game, free for educational use, involving Rube Goldberg inspired puzzles using simple physics-based interactions among objects. The simple premise of the game is seen as an advantage because it minimizes the importance of domain specific knowledge. This project specifically poses a series of puzzles of increasing difficulty to participants intermixed with interviews to better understand the problem solving process generally and specifically within the game. Video recordings of the computer screen allow study of the diverse ways in which participants solve puzzles and retrospective interviews of participants allow us to understand the degree to which individuals exploited structural similarities between puzzles to expedite 
problem-solving. Because this paper is a feasibility study, we will thoroughly describe what we did, the rationale behind it, our reflective evaluation of the effectiveness and viability of a more in-depth study, and next steps from the work.

\section{Pilot Study Methods}

After receiving IRB approval for human subjects research, we recruited five participants from the first-year general engineering courses at our institution. Through our reserach protocol we posed progressively more complex puzzles in Contraption Maker while also conducting a set of in-depth interviews before puzzle-solving, in-between select puzzles, and at the end of all puzzle-solving. The whole puzzle and interview protocol took approximately 1.5-2 hours per participant with about half of the time used for solving puzzles and the other half for interviews. Participants were given a $\$ 15$ gift certificate for their participation in the study. We used a screen capture utility to record both the screen and the audio from the internal microphone throughout the interview and activities. Once we had a file containing video and audio for each session a member of the research team skimmed each one to create a file containing time codes separating each interview and activity portion. We then used a small python program (available at https://github.com/hazybluedot/ffmpeg_split) to read the timecode file and execute the ffmpeg utility (https: / /wWw. f fmpeg.org/) to split the file into separate audio-only files for each interview portion and separate video-only files for each activity portion. Another member of the research team then transcribed each of the audio files and each transcription was sent to the corresponding participant to review for accuracy. To provide a more detailed look at these specific procedures, separate sections describing Contraption Maker and Problem-Solving Strategy Interviews are described in the text that follows.

\section{Contraption Maker}

Contraption Maker is both a commercial and educational game (i.e., can be purchased for 6.99 but is also free for teachers, schools, and STEM after school programs for educational use). The game's Educational Hub webpage (http: / / contraptionmaker.com/education/) documents various testimonials, shares possible curriculum mapped to Next Generation Science Standards, and even includes links to some research on the effectiveness of Contraption Maker's conceptual predecessor, The Incredible Machine, in the classroom. The critical role of sparking motivation and engagement with problem-solving in a STEM context, encouraging intellectual perseverance, and enabling students to learn coding to modify the game itself is not to be overlooked or diminished. However, research on what specific knowledge is gained and the transferability of that knowledge to other domains is understandably tougher to document but nonetheless of critical importance. For many educational games, claims of such learning gains are more rhetoric than the result of rigorous research. Other physics-based games such as Newton's Playground have been used to explore learning of physics content ${ }^{21}$ but lack the slick engaging

environment offered by Contraption Maker which, according to its own marketing, 22 "gives you a sandbox full of physics objects to build ridiculous chain reactions that do simple tasks in overly 
complicated ways." In fact, the driving feature leading us to explore Contraption Maker is the sandbox environment itself through which we can develop innovative puzzles ranging in structuredness and complexity supplemented by the developer's own puzzle bank. For reference, a screenshot of a basic puzzle is shown below (image was captured during this pilot study):

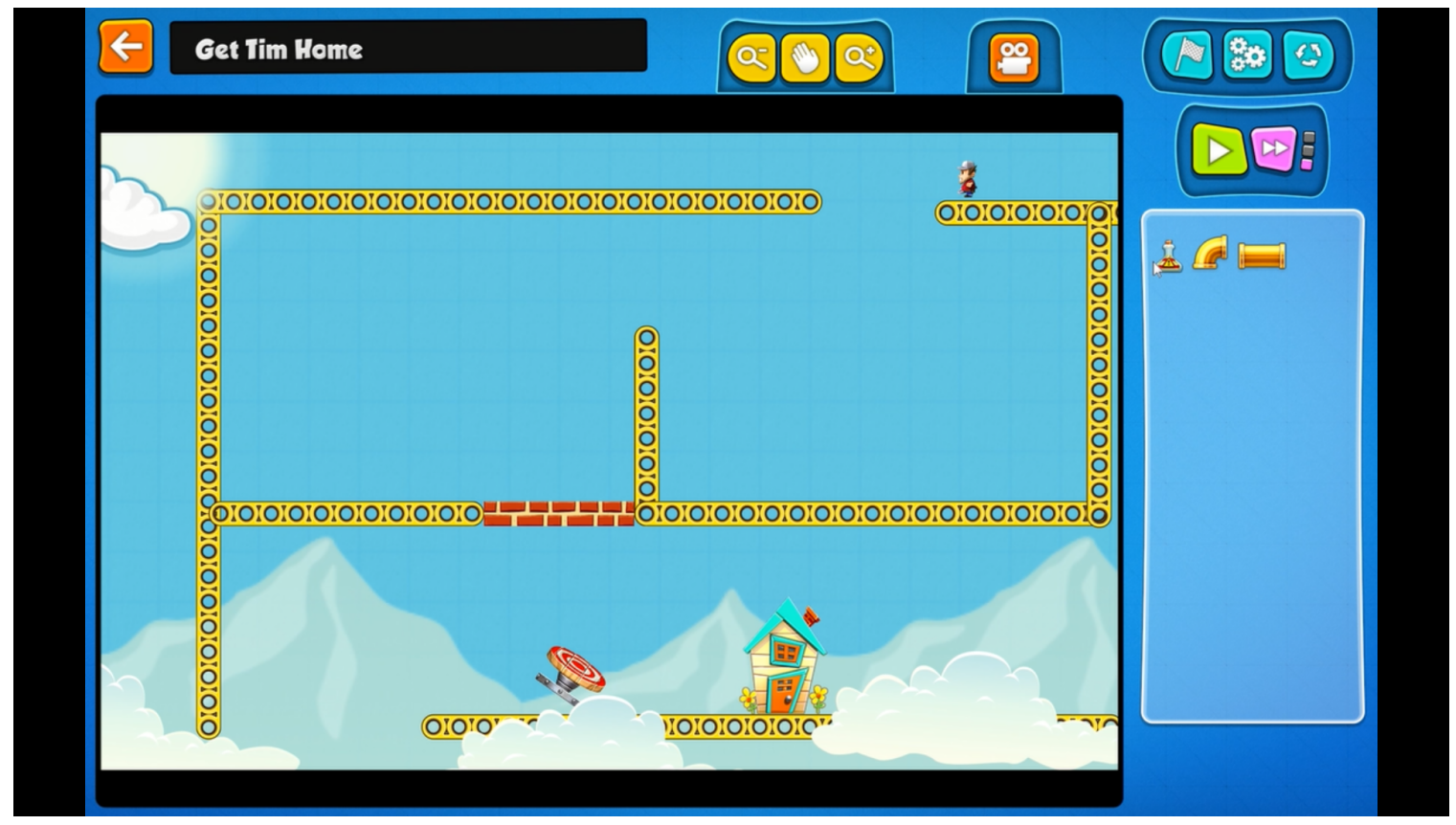

Figure 1: Screenshot of Contraption Maker Puzzle

All puzzles are characterized by a stock set of fixed items in the main field along with a palette of potential tools/items on the right-hand side to be used by the player. Once in the field, each object has a question mark query button that, when clicked, will provide very basic information about the object itself. The contraption can be run or reset using the green "play" button and the goal of the puzzle can be checked at any time by clicking on the flag button, both buttons are positioned at the top right of the screen. The example shown here is an easy puzzle with relative difficulty being a function of both (1) the number of components or interactions needed to achieve the goal state from a given start state and (2) the number (if any) of extra unnecessary pieces designed to distract in a players toolbox palette.

For reference, the specific slate of puzzles used in this pilot study along with timing of interviews are listed below:

\section{General Interview on Problem-Solving Strategies}

\section{Easy Puzzles}

- Easy Does It: Sibling Harmony

- Easy Does It: Fly Away Blimp

- Piece of Cake: Get Tim Home 
- Think Aloud Interview 1

\section{Medium Puzzles}

- Middle of the Road: Cannonball!

- I Like a Challenge: Excavation Site

- Think Aloud Interview 2

\section{Hard Puzzles}

- Pretty Much Impossible: Final Countdown

- Think Aloud Interview 3

\section{Reflective Interview on Experience and Connections to Strategies or Coursework}

Considering these puzzles and the playful environment, mapping specific content knowledge to a puzzle (e.g., physics conceptual knowledge as in the Newton's Playground study ${ }^{21}$ ) would be a challenging argument to justify. Thus, we are neither exploring the effectiveness of Contraption Maker as teaching tool in classrooms nor its ability to teach physics or other domain specific content. However, success on the puzzles requires the player to employ the same general problem-solving strategies (i.e. heuristics) used in much more advanced problems and discussed in problem-solving literature. Specifically, casting a complex problem as an amalgam of smaller more manageable chunks, successfully identifying which features are and are not worth one's attention, and overcoming functional fixedness of a specific object for creative insight. ${ }^{23 \mid 24}$ It is at this intersection between the simple electronic puzzle playing environment and classical problem-solving research that we see a critical opportunity. Because we can capture screenshot videos of the puzzle solving, we can employ learning analytics and mouse-click approaches to video coding (e.g., the work of ADAGE ${ }^{25}$ ) to track specific meaningful behaviors such as the number of times a player clicks the query button for objects (information gathering to define the problem and plan) or to capture the rapid acceleration of object placement that could indicate the "aha moment" of insight in discovering the solution and quickly implementing. By pairing such data with think-aloud interviews, we can corroborate or refute such coding in order to explore metacognitive activity in problem solving. That is, through such procedures we hope to not only observe and document specific strategies being used, or to hear the participant claim to use such strategies, but to see if and when both happen together.

\section{Interviews}

The semi-structured interviews were designed to get a sense of (1) how participants specifically conceptualize problems and problem-solving both generally and specific to the Contraption Maker puzzle environment and (2) to discuss how domain general problem-solving strategies are and are not connected to specific challenges within engineering disciplines. The interview protocols were open-ended in nature so that questions do not unintentionally frame the response. ${ }^{26}$ Interview questions to guide the conversation are given here.

\section{Part 1: Pre-Activity}


1. Please tell me about yourself and some of the experiences that led you to pursue a degree.

2. What specific activities or experiences do you think of when you hear "problem-solving"?

3. Do you consider yourself a good problem solver? Why or why not?

4. When faced with a problem, how do you proceed? When do those strategies change, if at all?

5. Tell me about a time when you encountered a problem and you weren't sure where to begin. How did you proceed? What motivated you to try that?

Part 2: During-Activity (repeated after specific puzzles as described earlier)

1. Talk me through your thought process on that puzzle... what did you do and why?

2. Did you find it particularly challenging? Why or why not?

3. What was going through your mind as you tried to work through it?

4. If solved: what were the key insights? If not: when/why did you give up?

\section{Part 3: Post-Activity}

1. Tell me about how you felt throughout the puzzle-solving experience.

2. Did you find yourself using different strategies for different puzzles?

3. How did you choose between strategies?

4. Did you find yourself using any of the problem solving strategies you described to me earlier while working through puzzles in Contraption Maker?

5. Now, I want you to think about problem solving in your engineering classes. Tell me about some of the problems you have to solve in a particular class. How would you describe those problems? What makes them hard or easy? What strategies do they require?

6. Compare and contrast working through problems in engineering classes and working through the puzzles in Contraption Maker

Given the exploratory nature of this study, findings from the interviews of five individuals are necessarily limited and thus should be used to only to inform and guide future studies rather than to draw significant conclusions about the topic itself. All interviews for each individual were transcribed verbatim and shown to study participants in order to verify the accuracy of the transcription process. In accordance with norms of qualitative research, 27 an inductive coding approach was employed in order to establish themes after the examination of the data with the specific goal of capturing the structures and processes described by study participants during the interviews. The lead analyst used ShareLaTeX to code data and vet themes with the collaborating researchers. First cycle coding of the interview data resulted in 27 distinct descriptions of the problem-solving process and experience. From these descriptions, two main themes emerged: strategies that the participants followed as they worked through the puzzles, and the experiences that they went through. The term "strategies" is defined operationally in this analysis as the steps and techniques that the participants employed while attempting to solve the puzzles that were 
provided to them, and articulated during the interviews. The term "experiences," on the other hand, is being used here to refer to the realizations, reactions, and responses that the participants expressed when talking about the various situations that they encountered and the outcomes of their efforts while engaged in the Contraption Maker activities.

\section{Strategies}

In talking about strategies, all participants recounted employing several problem-solving strategies both in the context of solving the Contraption Maker puzzles and in solving problems in general. One strategy in particular emerged as a common strategy across all participants, and was mentioned several times within the same interview by each participant: trial and error. While they verbally expressed the strategy in various ways, these expressions all pointed to an iterative process of trying out different variables or options until the task is accomplished or a solution is reached. An example of such a statement would be the following response that Amy shared when asked about her thought process after attempting to solve the Medium Puzzles: "I ran it through a couple of times just to figure out like what everything I was doing. So the trial helped me out like what's not happening that was happening in the first puzzle that's not happening now."

Bob expressed the same thought process in this manner, after completing the Easy Puzzles portion of the activity: "I just like to try all sorts of stuff until I find out what - what works." Later on, however, Bob explicitly names the strategy as trial and error: "My approach was simply just was just trial and error: if I do this, what happens." Sterling described his thought process as follows: "I just kept experimenting around and so I just went a bit far right, or attach it to something else. And then that would work."

The same thought process was shared by Tom, who said: "Um basically, does this work? No. Would this work? No. So basically just trying one thing, seeing how it would fit and if it didn't work, try something else." Finally, Anthony's response provided a more structured description of trial and error as a strategy, although the description still refers to an iterative process that involves trying different options: "I try to see what the best - method of solving it is and then I try it out to see if it works and if it does work then I'm done, but if it doesn't, come up with the next best method and then I keep going until I finally solve it."

The emergence of trial and error as the most common strategy for approaching problem-solving among the study participants was an interesting indicator of how students perceive problem-solving at this particular point in their academic careers: at the starting point of studying engineering. Jonassen ${ }^{11}$ identified troubleshooting and diagnosis as "among the most common types of problem solving," and trial and error as one of five global strategies commonly used in the troubleshooting process. Jonassen further described the trial and error as "randomly attack[ing] any section of the system where the possible fault might have occurred," which aligns with the operational definition for this descriptor as used in the analysis (an iterative process of trying out different variables or options until the task is accomplished or a solution is reached). In future work, strategies, and more specifically, trial and error, are both supported by literature and have shown up in this analysis and should be used as part of future analysis protocols. 


\section{Experiences}

The data likewise revealed shared experiences among the participants, despite the fact that each of the five sessions were conducted independent of each other. The most common experiences that emerged were that of experiencing the unknown, and of having to deal with increasing levels of difficulty that all participants associated with an increase in the number of variables or options that they could choose from. Participant experiences of the unknown were primarily expressed through questions that they posed to themselves, like "How do you do this?" or "How do I get this to work?" (Amy), or through explicit statements of not knowing or unfamiliarity, like "I had no clue" (Tom), "I'd never used a control system before" (Anthony), or "It's a game I've never played before" (Bob). Some experiences of the unknown and of unfamiliarity also flowed into experiences of increased level of difficulty. Bob, for example, shared: "So the first puzzle that I tried to solve was the hardest because I didn't really know what I [was] doing because it's a new game and I need to get used to - to the interface because it's something that's unfamiliar to me." Since the activities were structured in increasing levels of difficulty, participant experiences invariably expressed this occurrence.

The analysis further revealed that participants associated increased level of difficulty with the number of variables or options present towards arriving at a solution to the problem. Generally, participants expressed that the more variables or options are present, the more difficult it was to solve the puzzle in Contraption Maker, or any problem in general. They expressed these sentiments as follows, sometimes including the experience of the unknown or unfamiliarity as a contributing factor to the increased level of difficulty:

AMY: "I guess what makes something hard is having so many options and not knowing where to start versus something easy where it's, 'oh, there's only three choices here, let's try them each and we'll figure it out."'

BOB: "I was quite overwhelmed by all of the new pieces that were involved in the game board."

ANTHONY: "The easier ones I thought were the ones with less items and less, like, complex um, obstacle course I guess. So yeah.”

STERLING: "There were very few variables." (When asked what made a task simple)

TOM: "For me, it's really just the amount of variables that I don't see or the amount of chaos that's going on or a starting point." (When asked what makes a task hard or easy)

This data is particularly interesting in the context of other comments where participants described what makes problems "easy" or "hard" and how to approach such problems. Bob associated the level of difficulty of a puzzle or problem with the amount of time that was given to solve it, or that was required to actually complete it: "It's not exactly how hard it is, but how long it takes." In addition, Bob also talked about using prior knowledge or experiences in solving current problems: "So like stuff from past problems can be used to solve future problems." Anthony provided descriptions of a strategy that involved breaking a problem down into more manageable pieces. He shared: "I like to break things down also into like easier steps and I keep working 'til I can solve something." Tom, on the other hand, also described a problem-solving process that involved a "sequence of steps." He talked about starting simple, and building up from there: 
"Really I look to see what is the simplest method and how can I improve and justify the method that I'm using. I usually look for the simplest way and basically just build on top of that."

Collectively, this analysis of the experience of problem-solving suggests future problem-solving protocols should involve puzzles with varied difficulty according to several metrics (e.g., number of choices, number of items/variables, amount of time to solve). More formally in the literature, such difficulty is referred to as the complexity of the problem as contrasted to the dynamicity which involves changing problem formulation or goals over time such as in complex engineering design tasks. 11 Additionally, future analysis should focus on any alignment or misalignment between (1) what a participant claimed made a problem challenging, (2) the problem formulation itself and what was technically required for a solution, and (3) whether any correlations exist between the perception of the problem and the individual's success in solving it.

\section{Video Coding}

At the outset, we sought to leverage the capabilities of computers to track movements and mouse-clicks in order to study behavioral measures that could be used to corroborate data coming from think aloud interviews. Such methods of analysis from educational computer games have been documented (e.g., the work of ADAGE ${ }^{25}$ ) and some educational games can provide this service of tracking telemetry data in the system (e.g., SimCityEDU

https: / / www.glasslabgames.org/games/SC. However, a means of automatically collecting such data from the game itself is not feasible with Contraption Maker because it was not designed with that purpose in mind. However, we can easily capture video recordings of the screen during the problem-solving activity and post-process. While watching a video, a researcher can easily document important metrics such as if a puzzle was solved or not and the time taken to solve the puzzle. As we explored the video data, we noted that certain behaviors might help us better understand specific phenomenon of interest but that such analysis is time intensive because it involves researchers re-watching every minute of data. Thus, for the purpose of this feasibility study, we have identified specific metrics that should be studied throughout video data along with logic for operationalizing and automating such behavior:

\begin{tabular}{||c|c|c||}
\hline Problem-Solving Behavior & Triggering Events & Notes for Automation \\
\hline \hline Success in Solving Problem & Puzzle Complete Pop Up & Study Repeatable Pixel Color Changes \\
\hline Duration of Attempt & Pop Ups for Start and Stop & Count Time Between Pop Ups \\
\hline Help Seeking Behavior & Clicking ? for an Item & Study Pixel Changes with Pop Up \\
\hline Number of Attempts & Clicking Play Button on Machine & "Play" Changes Shape and Color \\
\hline
\end{tabular}

All the metrics above can be automated through conversion of video data to frames of pixel data and coding in the specific behaviors to track and count (e.g., change in shape and color of the "play" button to count attempts). Specific data for each behavior and each individual number are not shown for this feasibility study - instead our purpose here was to identify behaviors, trigger events, and possible means of automating the analysis. Several other metrics were identified but the process to automate will require further research and study. For example, in watching video data, our team noticed that for some individuals and puzzles, there were moments of insight 
which were characterized by a shift in seemingly random placement of objects with no success to sudden intentional rearranging leading to a solution. As an analogy, consider a crossword puzzle or Sudoku puzzle where filling in a critical clue or box leads to a rapid conclusion.

Such behavior was noted in this study but writing code to automate this feature is outside of the current project scope. The open source computer vision library OpenCV

(http: / / opencv.org) seems a promising fit: it can be used to track specific obects and motion. With these more advanced techniques we could recognize different Contraption Maker objects and detect when a player places them from a pallete, when they are moved, and calculate an associated distance-from-correct-position over time to approximate a sudden "ah-ha" moment, when several objects that were not correctly placed are quickly moved to their solution positions in rapid succession. Admittedly, developing these more advanced techniques will be challenging and they will need to undergo extensive validity testing, but we are optimistic that they will eventually allow us to easily analyze batches of screen capture video to detect patterns across participants and puzzles well beyond what has been demonstrated in this current study (i.e., basic behaviors such as number of attempts).

Once automated, this study can be scaled up to include sufficient participants so that quantitative analyses could be performed to study systematic differences across groupings (e.g., does help seeking behavior have an impact on success in solving problems?)

\section{Reflective Discussion and Next Steps}

As a feasibility study, the work shows promise but findings remain limited. We were pleasantly surprised at the engagement and persistence of the participants in the study (several did not want to give up on the hardest puzzles, worked beyond the allotted time for the puzzles, and even talked about being both frustrated and having fun in the same sentence). Anecdotally, this reinforces our hopes at the outset of the study - that is, that by using a game, our participants continue to display the persistence that we feel would be needed to most accurately study problem-solving in increasingly complex situations.

Further, the interview protocols functioned as designed and did elicit meaningful data from participants. Results from coding of interviews from the five participants identified specific codes that should be used to inform the coding of a future study with a larger sample.

Similarly, video recorded data was collected and we were able to identify specific behaviors to be tracked as well as means of automating analysis for some of the behaviors. This piece is especially critical in order to scale up the study in the future.

One significant component of future work not demonstrated here is to create individual cases from the aggregate data for each participant and to conduct a multiple case-study analysis across the participants. Such future analysis will allow for us to (1) study the degree to which data from across the methods aligns (e.g., participant claims trial and error methods and demonstrates them in the video data) and (2) compare and contrast across cases to develop more cross-cutting insights about problem solving phenomenon. 
Thus far, this proof of concept work has focused on problem-solving strategies generally and the use of stock puzzles from the game. However, as discussed at the outset, a primary motivator for evaluating this tool was that we believe it might be useful in isolating and studying analogical reasoning. Of specific interest would be if we could find a way to meaningfully study (1) if and when novel problems are framed using similar analogous problems and (2) the degree to which problem-solvers actively regulate their use of analogical reasoning. Our initial work thus far with

Contraption Maker suggests that we might develop a slate of puzzles specifically designed so that particular puzzles might be solved at a more rapid pace if the problem-solver recognizes and exploits a specific connection (i.e., a mapping of structural similarities from one analogous previously-solved problem to a more ill-structured and novel problem at hand). We propose intermingling the puzzles specifically designed for this purpose with select stock puzzles from the game to serve as placebo puzzles. Interview protocols could follow the same procedures as outlined in this study. We believe this work to be challenging but feasible. While Contraption Maker offers full functionality to create our own puzzles in its Maker Space, and we can even use JavaScript to mod basic code associated with objects to achieve additional flexibility, the challenge remains in creating the interrelated set of puzzles themselves. Incorporating embedded analogous elements throughout puzzles in ways that are structurally similar but not immediately obvious to the player is difficult but worthwhile and ongoing work.

\section{References}

[1] Lisa R. Lattuca, Linda C. Strauss, and J. Fredericks Volkwein. "Getting in Sync: Faculty and Employer Perceptions from the National Study of EC2000”. English. In: International Journal of Engineering Education 22.3 (June 2006), pp. 460-469.

[2] National Academy of Engineering NAE. The Engineer of 2020: Visions of Engineering in the New Century. Washington, D.C.: National Academies Press, May 2004.

[3] Educate to Innovate: Factors That Influence Innovation: Based on Input from Innovators and Stakeholders. Washington, D.C.: National Academies Press, Apr. 2015.

[4] Mirka Koro-Ljungberg et al. "Reconceptualizing and decentering think-aloud methodology in qualitative research”. en. In: Qualitative Research 13.6 (Dec. 2013), pp. 735-753. DOI: $10.1177 / 1468794112455040$.

[5] Lisa Benson and David Bowman. "Museink: Seeing And Hearing A Freshman Engineering Student Ink And Think". In: June 2010, pp. 15.898.1-15.898.7.

[6] David H. Jonassen. "Toward a design theory of problem solving". en. In: Educational Technology Research and Development 48.4 (Dec. 2000), pp. 63-85. DOI: 10.1007 / BF 02300500 .

[7] Namsoo Shin, David H. Jonassen, and Steven McGee. "Predictors of well-structured and ill-structured problem solving in an astronomy simulation”. en. In: Journal of Research in Science Teaching 40.1 (Jan. 2003), pp. 6-33. DOI: 10.1002 /tea.10058.

[8] J.A. Meacham and N.M. Emont. "The interpersonal basis of everyday problem solving". en. In: Everyday problem solving: theory and applications. Ed. by Jan D. Sinnott. Praeger, Jan. 1989.

[9] David H. Jonassen. "Instructional design models for well-structured and III-structured problem-solving learning outcomes”. en. In: Educational Technology Research and Development 45.1 (Mar. 1997), pp. 65-94. DOI: $10.1007 / \mathrm{BF} 02299613$. 
[10] Schön. "The Design Process". en. In: Varieties of thinking: essays from Harvard's Philosophy of Education Research Center. Ed. by V. A. Howard. Routledge, Apr. 1990.

[11] David H. Jonassen. Learning to Solve Problems: A Handbook for Designing Problem-Solving Learning Environments. en. Taylor \& Francis, Sept. 2010.

[12] Charoula Angeli and Nicos Valanides. "Epistemological Beliefs and Ill-structured Problem-solving in Solo and Paired Contexts". In: Journal of Educational Technology \& Society 15.1 (2012), pp. 2-14.

[13] Gregory Schraw, Michael E. Dunkle, and Lisa D. Bendixen. "Cognitive processes in well-defined and ill-defined problem solving”. en. In: Applied Cognitive Psychology 9.6 (Dec. 1995), pp. 523-538. DoI: $10.1002 /$ acp.2350090605.

[14] Cynthia J. Atman and Karen M. Bursic. "Verbal Protocol Analysis as a Method to Document Engineering Student Design Processes". en. In: Journal of Engineering Education 87.2 (Apr. 1998), pp. 121-132. DOI: $10.1002 / j .2168-9830.1998 . t b 00332 . x$

[15] K. Anders Ericsson and Herbert A. Simon. "Verbal reports as data." en. In: Psychological Review 87.3 (1980), pp. 215-251. DOI: $10.1037 / 0033-295 X .87 .3 .215$.

[16] Edward M. Sosu. "The development and psychometric validation of a Critical Thinking Disposition Scale". en. In: Thinking Skills and Creativity 9 (Aug. 2013), pp. 107-119. DoI: $10.1016 /$ j.tsc.2012.09.002

[17] Karl Duncker. “On problem-solving”. en. Trans. by Lynne S. Lees. In: Psychological Monographs 58.5 (1945), pp. i-113. DOI: $10.1037 /$ h0093599

[18] James Paul Gee. "Learning by Design: good video games as learning machines". en. In: E-Learning 2.1 (2005), p. 5. DOI: 10.2304 /elea.2005.2.1.5.

[19] Gee. Are video games good for learning? Feb. 2007.

[20] R.J. Mislevy et al. "Psychometrics and Game-Based Assessment". en. In: Technology and Testing: Improving Educational and Psychological Measurement. Ed. by Fritz Drasgow. Routledge, Aug. 2015.

[21] Valerie J. Shute, Matthew Ventura, and Yoon Jeon Kim. "Assessment and Learning of Qualitative Physics in Newton's Playground". In: The Journal of Educational Research 106.6 (Nov. 2013), pp. 423-430. DOI: $10.1080 / 00220671.2013 .832970$

[22] Contraption Maker - Play. Make. Learn.

[23] Robert E. Adamson. "Functional fixedness as related to problem solving: a repetition of three experiments." en. In: Journal of Experimental Psychology 44.4 (1952), pp. 288-291. DOI: $10.1037 /$ h0 062487.

[24] Herbert A. Simon. The role of attention in cognition. New York: Academic Press, 1986.

[25] V. Elizabeth Owen et al. "Capturing Learner Trajectories in Educational Games through ADAGE (Assessment Data Aggregator for Game Environments): A Click-Stream Data Framework for Assessment of Learning in Play”. In: Annual Meeting of the American Educational Research Association, Philadelphia, April. 2014, pp. 3-7.

[26] Gretchen B. Rossman and Sharon F. Rallis. Learning in the Field: An Introduction to Qualitative Research. en. SAGE, July 2011.

[27] Johnny Saldana. The Coding Manual for Qualitative Researchers. en. SAGE Publications, Feb. 2009. 